\title{
MICROARRAYS
}

\section{DEALing with protein microarrays}

A new method for attaching antibodies to a substrate offers a simple and reliable means of constructing protein microarrays.

DNA arrays are powerful tools for the क analysis of gene expression because of the high level of multiplexing they provide. Researchers have adapted this technology for protein detection by arraying antibodies but challenges in the creation and stability of protein arrays make them difficult to use. James Heath of the California Institute of Technology and colleagues describe a method for constructing DNA-encoded antibody libraries, or DEAL, that solves this problem.

Heath says, "My post doc, Ryan Bailey, came up with this idea of trying to have one common chemistry based on DNA. We pushed it pretty hard from there, and it ended up working extremely well."

DNA provides two important properties. 을 First, it is very stable and easily immobilized on convenient substrates. Second, the use of complementary ssDNAs provides the perfect platform for spatial multiplexing. "DNA provided a self assembly technique that had high encoding power because for each additional nucleotide you add $4^{n}$ encoding power," remarks Bailey, who now has his own lab at the University of Illinois.

Briefly, ssDNAs are spotted on an array, and antibodies are conjugated to complementary ssDNAs. The tagged antibodies are incubated with a sample solution, and the mixture is placed on the surface of the chip for detection. Not only can a variety of antibodies be multiplexed, but other sensors can also be easily incorporated on the same chip.

Unlike protein arrays, DNA arrays can withstand the fabrication process used in constructing microfluidic devices, allowing integration of the two technologies. Microfluidics speeds up analysis because flowing the analyte through a microfluidic channel on a DEAL-based array avoids dif- fusion limitations and facilitates multiplexed detection. "We've extended protein detection to 10 parallel assays in a single channel," says Bailey. "More advanced microfluidics would add higher levels of functionality and automation, but we haven't pushed in this direction."

Although many cutting-edge laboratory techniques tend to be difficult to apply or not very robust, Heath says this is not the case for DEAL. "In my lab now we've trained pathologists and biologists as well as regular chemistry and bioengineering graduate students." This suggests that other labs should be able to apply DEAL quite easily, which should be a good deal for everyone.

\section{Daniel Evanko}

\section{RESEARCH PAPERS}

Bailey, R.C. et al. DNA-encoded antibody libraries: a unified platform for multiplexed cell sorting and detection of genes and proteins. J. Am. Chem. Soc. 129, 1959-1967 (2007). 